\title{
Application of Radial Point Interpolation Method to Temperature Field
}

\author{
Yu Chen, Maohui Xia, Dehua Wang \& Dongmei Li \\ College of Sciences, Yanshan University \\ Hebei 066004, China \\ E-mail: chenyu3025@126.com
}

\begin{abstract}
A point interpolation which bases on the radial function is a new meshless method. It is advantageous over the original PIM with polynomial basis in avoiding singularity when shape functions are constructed. It is also easy to deal with essential boundary for its property of Kronecher Delta function. To verify it's valid, this paper introduced the basic principle of RPIM. In addition, numerical example of heat conduction showed that the new methods possessed several advantages, such as high efficiency, high accuracy, and high stability. It is a promising method in physics.
\end{abstract}

Keywords: Meshless methods, Point interpolation methods, Radial basis functions, Radial point interpolation method

\section{Introduction}

Meshless method is a new numerical analysis method which has rapidly developed in recent years (Zhang Xiong and Liu Yan, 2004). Because it bases of nodes information and thoroughly or partially eliminates the grids, and it also has high efficiency, easy to implement, therefore it is more flexible and effective in dealing with large deformation, high-gradient and other advantages than the traditional finite element.

Recent meshless approximations mainly have the following types of programs (LIU G.R, 2007), element-free Galerkin method (EFGM), local Peter-Galerkin method (MLPG), Point Interpolation (PIM) and so on, but mostly shape functions don't have Kronecker Delta function property, which makes the essential boundary conditions hard to deal with. Radial Point Interpolation Method (RPIM) is a new meshless method (XIONG Yuan-bo, LONG Shu-yao and LIU Kai-yuan, 2007, p135-138. XIA Mao-hui , JIA Yan and LIU Cai, 2006, p112-117), its shape function is constructed by the combination of radial and polynomial basis functions. Because it has the property of delta functions, so it is convenient to implement the boundary conditions. At the same time its interpolation process is very similar to the finite element, so a lot of finite element procedures can be applied directly. Select the appropriate shape parameters is the key to Radial point interpolation method, which is usually determined by empirical formula.

At present, meshless method appling to the temperature field mainly use EFGM(GAO Zhi-hua, ZHANG Ming-yi and LIU Zhi-qiang, 2006, p545-550. YUAN Su-ling, GE Yong-qing and WANG Zhang-qi, 2003, p82-86) and MLPG (LI Qing-hua, CHEN Shen-shen and XIONG Yong-gang, 2006, p22-24). This article introduced the basic theory of the radial point interpolation (RPIM), and used this method to construct the interpolation function, and applied it to two-dimensional steady-state temperature field, and example verified that RPIM is a less time-consuming and high precision, simple and effective computing method.

\section{Point Interpolation Method}

Consider a scalar function $u(x)$ defined in problem domain $\Omega$, set several nodes in and on the domain randomly, use total of $n$ field nodes included in the local support domain $\Omega$ of the point of interest at $x_{q}$ to interpolate, and the $u(x)$ at $x_{q}$ is approximated in the form of

$$
u^{h}\left(x, x_{q}\right)=\sum_{i=1}^{n} R_{i}(x) a_{i}+\sum_{j=1}^{n} P_{j}(x) b_{j}=R^{T}(x) a+P^{T}(x) b
$$

Where $R_{i}(x)$ is a radial basis function, $P_{j}(x)$ is monomial in the space coordinates $x^{T}=\{x, y\}, m$ is the number of polynomial basis functions. Coefficients $a_{i}, b_{j}$ are constants yet to be determined. We also choose $m<n$ to have better stability of the interpolating function. In two-dimensional problems, the general linear-based $P^{T}(x)=[1, x, y]$ is used. 
For given $x$, we have

$$
\begin{gathered}
a=\left[a_{1}, a_{2}, \cdots, a_{n}\right]^{T} \\
b=\left[b_{1}, b_{2}, \cdots, b_{m}\right]^{T} \\
R^{T}(x)=\left[R_{1}(x), R_{2}(x), \cdots, R_{n}(x)\right] \\
P^{T}(x)=\left[P_{1}(x), P_{2}(x), \cdots, P_{m}(x)\right]
\end{gathered}
$$

Typically, in two-dimensional problems

$$
\begin{gathered}
R_{i}(x)=R_{i}\left(r_{i}\right)=R_{i}(x, y) \\
r_{i}(x)=\left[\left(x-x_{i}\right)^{2}+\left(y-y_{i}\right)^{2}\right]^{1 / 2}
\end{gathered}
$$

Now enforcing equation (1) to be satisfied at nodes to determine the coefficients $a_{i}, b_{j}$, the matrix form is

$$
U_{S}=R_{Q} a+P_{m} b
$$

Where $U_{S}=\left[u_{1}, u_{2}, \cdots, u_{n}\right]^{T}$, Matrix $R_{Q}$ is given by

$$
R_{Q}=\left[\begin{array}{cccc}
R_{1}\left(x_{1}\right) & R_{2}\left(x_{1}\right) & \cdots & R_{n}\left(x_{1}\right) \\
R_{1}\left(x_{2}\right) & R_{2}\left(x_{2}\right) & \cdots & R_{n}\left(x_{2}\right) \\
\vdots & \vdots & \vdots & \vdots \\
R_{1}\left(x_{n}\right) & R_{2}\left(x_{n}\right) & \cdots & R_{n}\left(x_{n}\right)
\end{array}\right]
$$

The matrix $P_{m}$ is a $n \times m$ matrix given by

$$
P_{m}=\left[\begin{array}{cccc}
P_{1}\left(x_{1}\right) & P_{2}\left(x_{1}\right) & \cdots & P_{m}\left(x_{1}\right) \\
P_{1}\left(x_{2}\right) & P_{2}\left(x_{2}\right) & \cdots & P_{m}\left(x_{2}\right) \\
\vdots & \vdots & \vdots & \vdots \\
P_{1}\left(x_{n}\right) & P_{2}\left(x_{n}\right) & \cdots & P_{m}\left(x_{n}\right)
\end{array}\right]
$$

However, there are $n+m$ variables in equation (5), but only have $n$ equations, so it is an undetermined equations, solving the above equation (5) needs to impose a constraint equation

$$
P_{m}^{T} a=0
$$

Combing equations (5) and (8), the matrix form becomes

$$
\left[\begin{array}{cc}
R_{Q} & P_{m} \\
P_{m} & 0
\end{array}\right]\left\{\begin{array}{l}
a \\
b
\end{array}\right\}=\left\{\begin{array}{c}
U_{S} \\
0
\end{array}\right\}
$$

Solving equation (9), we can obtain

$$
\begin{aligned}
& b=S_{B} U_{S} \\
& a=S_{a} U_{s}
\end{aligned}
$$

Where $S_{b}=\left[P_{m}^{T} R_{Q}^{-1} P_{m}\right]^{-1} P_{m} R_{Q}^{-1}, S_{a}=R_{Q}^{-1}-R_{Q}^{-1} P_{m} S_{b}$.

Substituting $a, b$ back into equation (1), we obtain

$$
u^{h}\left(x, x_{q}\right)=\left[R^{T} S_{A}+P^{T} S_{b}\right] U_{S}=\sum_{i=1}^{n} \Phi_{i}(x) u_{i}=\Phi(x) U_{S}
$$

Where shape function $\Phi(x)$ is given by

$$
\Phi(x)=\left[\Phi_{1}(x), \Phi_{2}(x), \cdots, \Phi_{n}(x)\right]
$$

The derivatives of shape functions can be easily obtained as 


$$
\left\{\begin{aligned}
\frac{\partial \Phi_{k}}{\partial x} & =\sum_{i=1}^{n} \frac{\partial R_{i}}{\partial x} S_{i k}^{a}+\sum_{j=1}^{n} \frac{\partial P_{j}}{\partial x} S_{j k}^{b} \\
\frac{\partial \Phi_{k}}{\partial y} & =\sum_{i=1}^{n} \frac{\partial R_{i}}{\partial y} S_{i k}^{a}+\sum_{j=1}^{n} \frac{\partial P_{j}}{\partial y} S_{j k}^{b}
\end{aligned}\right.
$$

Here are three often used globally supported radial basis functions:

(1)Multi-quadrics(MQ):

$$
R_{i}(x)=\left(r_{i}^{2}+c^{2}\right)^{q}=\left[\left(x-x_{i}\right)^{2}+\left(y-y_{i}\right)^{2}+c^{2}\right]^{q} \quad c>0
$$

(2)Gaussian(EXP):

$$
R_{i}(x)=\exp \left(-c r^{2}\right)=\exp \left(-c\left[\left(x-x_{i}\right)^{2}+\left(y-y_{i}\right)^{2}\right]\right)
$$

(3)Thin plate spline:

$$
R_{i}(x)=r_{i}^{\eta}=\left[\left(x-x_{i}\right)^{2}+\left(y-y_{i}\right)^{2}\right]^{\eta} \quad \eta \in N
$$

In above equations, $c, q, \eta$ are all called shape-parameters, $r=\left\|x-x_{i}\right\|$. Normally, the choosing of these parameters will effect the result. J.G. Wang and G.R. Liu have discussed in their papers. They also discovered that $q=1.03, c=1.42$ performs best in MQ. We will use this result in the following example, the derivative of MQ function is:

$$
\left\{\begin{array}{l}
\frac{\partial R_{i}}{\partial x}=2 q\left(r_{i}^{2}+c^{2}\right)^{q-1}\left(x-x_{i}\right) \\
\frac{\partial R_{i}}{\partial y}=2 q\left(r_{i}^{2}+c^{2}\right)^{q-1}\left(y-y_{i}\right)
\end{array}\right.
$$

\section{Discretized equation of Temperature field}

Consider the issue of two-dimensional steady-state temperature field:

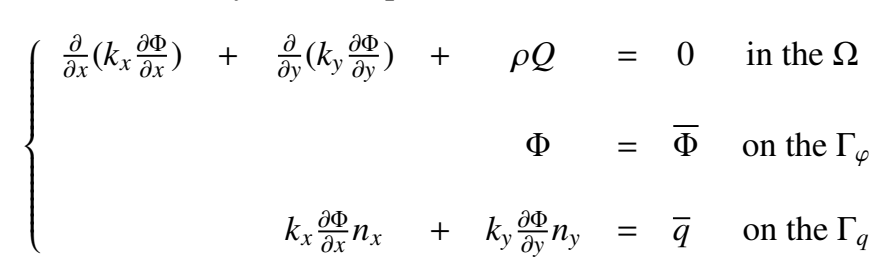

Where $\Phi$ indicates temperature, $\Gamma_{\varphi}$ is the Dirichlet border, $\Gamma_{q}$ is Neumann border, $\rho$ is material density, $k_{x}$ is the thermal conductivity coefficient along the direction of $x, k_{y}$ is the thermal conductivity material coefficient along the direction of $y, Q$ is the object density of internal heat source, $n_{x}$ and $n_{y}$ is the boundary normal direction cosine.

The standard variational (weak) form of equation (18) is posed as follows:

$$
F(A)=\int_{\Omega}\left[\frac{1}{2} k_{x}\left(\frac{\partial \Phi}{\partial x}\right)^{2}+\frac{1}{2} k_{y}\left(\frac{\partial \Phi}{\partial y}\right)^{2}-\Phi \rho Q\right] d \Omega-\int_{\Gamma_{q}} \Phi \bar{q} d \Gamma
$$

Substituting equation (11) back into equation (19), we can obtain

$$
K \Phi=F
$$

Where

$$
\begin{gathered}
K_{I J}=\int_{\Omega}\left(k_{x} N_{I, x} N_{J, x}+k_{y} N_{I, y} N_{J, y}\right) d \Omega \\
F_{I}=\int_{\Omega} N_{I}(\rho Q) d \Omega+\int_{\Gamma_{q}} N_{I} \bar{q} d \Gamma
\end{gathered}
$$

\section{Numerical example}

As shown in Figure 1, $5 \times 5$ square region, given constant temperature $\Phi=0^{\circ} \mathrm{C}$ on the edge of $x=0, x=5$ and $y=0$, along the edge of $y=5$ set a constant temperature of $\Phi=10^{\circ} \mathrm{C}$, with no heat source, thermal conductivity coefficients $k_{x}=k_{y}=1$. RPIM used to calculate the temperature distribution, the problem domain is represented by $225(15 \times 15)$ regularly distributed nodes, $14 \times 14$ rectangular Gaussian background cells are used for numerical interactions. In each background cell, $3 \times 3$ Gaussian points are employed. Compare RPIM solution to the exact solution on the cross section $x=2.5$, Figure 2 gives the trends of cross section on the meshless solution, exact solution and FEM solution. Through figure 2 we can see that the meshless results are basically consistent with the exact solution, which verified the accuracy and effectiveness of RPIM. 


\section{Conclusions}

(1). Meshless method only needs nodes of information and boundary conditions, which cast off the restricted units of the finite element method and decrease the work of finite element method in the complex mesh generation and re-dividing.

(2). RPIM method is more advanced than the element-free Galerkin method based on the mobile least squares, as long as $R_{Q}^{-1}$ determined, shape function and its derivative will be able to determined. Shape function possess $\delta$ function characteristics, it is easier to handle essential boundary conditions.

(3). This paper attempted to promote this method to the temperature field problem, example results show that using this method to deal with the issue of temperature field receives satisfactory results, which further validated the meshless method RPIM is accuracy and effectiveness.

\section{References}

Gao, Zhi-hua, ZHANG, Ming-yi \& LIU, Zhi-qiang. (2006). A Meshless Method for Nonlinear Transient Field with Phase Change.Hinese Journal of Computational Physics, 9, 545-550.

Liu, Gui-rong, GU, Yuan-tong, WANG, Jian-ming \& ZHOU, Xue-Jun. (2007). Meshless methods and program design theory. Jinan: Shandong University Press.

Li, Qing-hua, CHEN, Shen-shen \& XIONG, Yong-gang. (2006). A Local Petrov-Galerkin Method for Heat Conduction Problem. Journal of Zhuzhou Institute of Technology, 3, 22-24.

Xia, Mao-hui, JIA, Yan and LIU, Cai. (2006). A Meshless Method with Radial Interpolation Method (RPIM). Journal of Yanshan University, 3, 112-117.

Xiong, Yuan-bo, LONG, Shu-yao \& LIU, Kai-yuan. (2007). Analysis of Elaso- Plastic Problem by Meshless Method . Journal of Mechanical strength, 29 (1), 135-138.

YUAN, Su-ling, GE, Yong-qing \& WANG, Zhang-qi. (2003). Element-free method for temperature profile in heat conduction. Journal of North China Electric Power University, 3, 82 -86.

Zhang, Xiong \& Liu, Yan. (2004). Meshless method. Beijing, Tsinghua University Press.

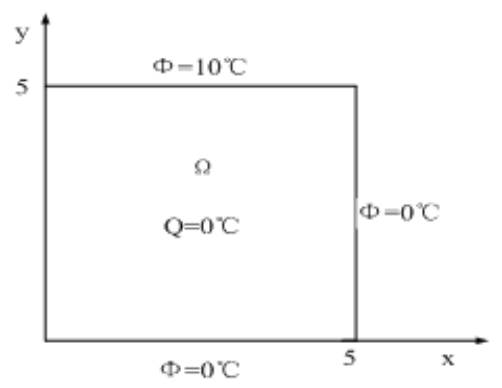

Figure 1. Heat conduction model of two-dimension

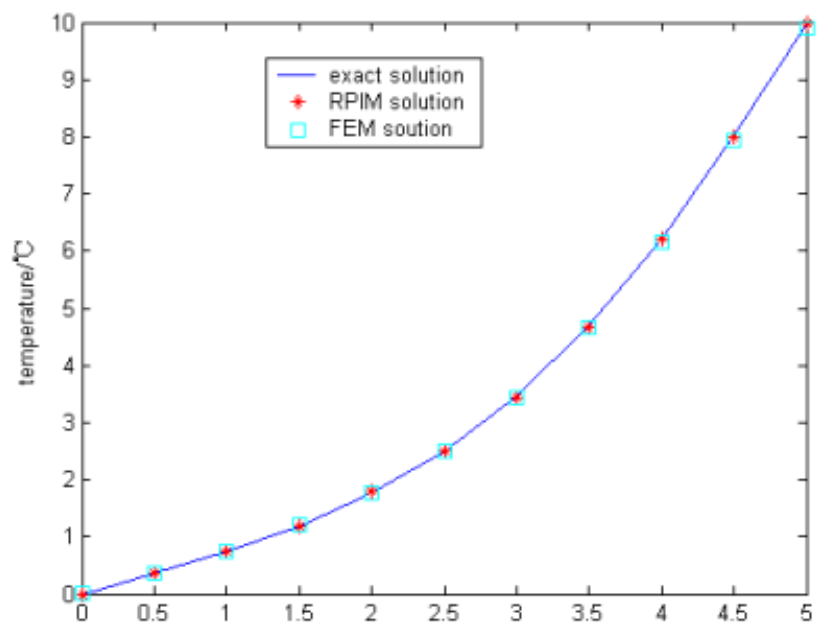

Figure 2. The change of temperature along $x=2.5$ 